Case Study

\title{
Polyostotic Fibrous Dysplasia of Ribs
}

\section{Bidyut Kumar Sahu}

MS (General Surgery), Head Consultant, TATA Central Hospital, West Bokaro, Jharkhand, India.

DOI: https://doi.org/10.24321/2394.6539.202111

I $\quad \mathbf{N} \quad \mathbf{F} \quad \mathbf{O}$

E-mail Id:

bidyut.sahu@gmail.com

Orcid Id:

https://orcid.org/0000-0003-2762-7244

How to cite this article:

Sahu BK. Polyostotic Fibrous Dysplasia of Ribs. J

Adv Res Med Sci Tech. 2021;8(3):13-15.

Date of Submission: 2021-04-20

Date of Acceptance: 2021-09-10

\section{$\begin{array}{llllllll}\mathbf{A} & \mathbf{B} & \mathbf{S} & \mathbf{T} & \mathbf{R} & \mathbf{A} & \mathbf{C} & \mathbf{T}\end{array}$}

34 yr old female presenting with pain lower part of left side chest was found to have incidental radiological finding which revealed a rare genetic condition of ribs.

Keywords: Polyostotic, Fibrous Dysplasia, Rib

\section{Introduction}

Chest pain over left side in an otherwise healthy young lady patient in OPD set up may be of varied aetiology. Having excluded cardiac origin pain, many other possibilities can be considered under differential diagnosis for noncardiac chest pain. Though often overlooked as myalgia or, somatisation disorder, sometimes a genetic condition like fibrous dysplasia can be a latent finding. A multi-osseous site involvement should alert the clinician for regular follow up, screening for McCune-Albright syndrome and occult bony metastasis.

\section{Case Presentation}

A $34 \mathrm{yr}$ old otherwise healthy female, not having any systemic illness presented in surgical OPD in 2010 with pain left side back, over lower part of ribs, of 15 days duration, more intense on movement and stretching. Upon examination, mild tenderness and swelling without any feature of local inflammation was noted. Thoraco-dorsal spine had no scoliosis, deformity or gibbus.

No major restriction of movement or, abnormality of gait was found. Pt. further revealed such painful episodes intermittently since early adolescence, relieved with analgesics. No h/o- fever, local trauma or, of ATT administration was found. Pt. returned 3 days later without relief with NSAID, hence further investigations were advised.

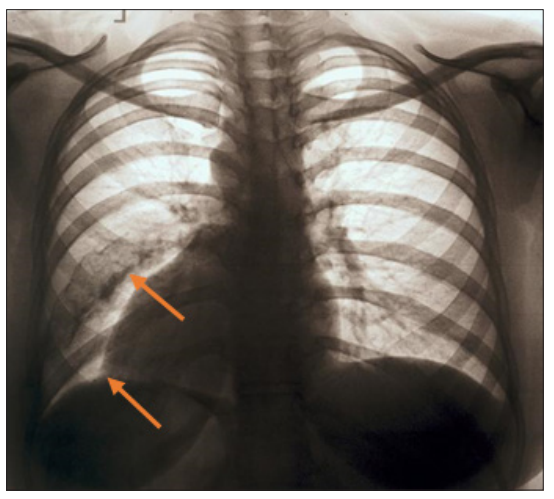

Figure I.Chest X-Ray Invert Contrast

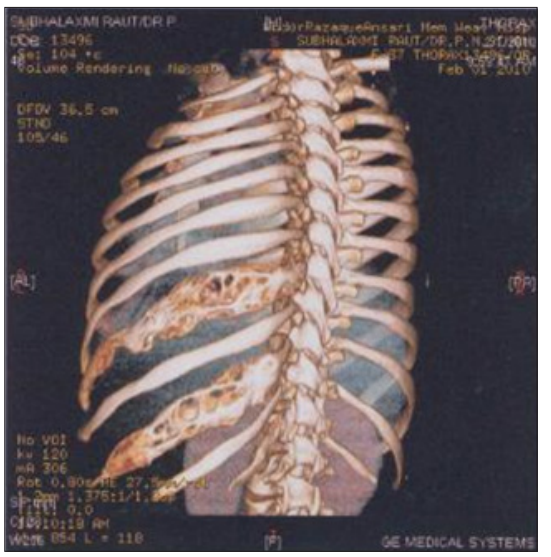

Figure 2.Axial CT Scan of Ribs 


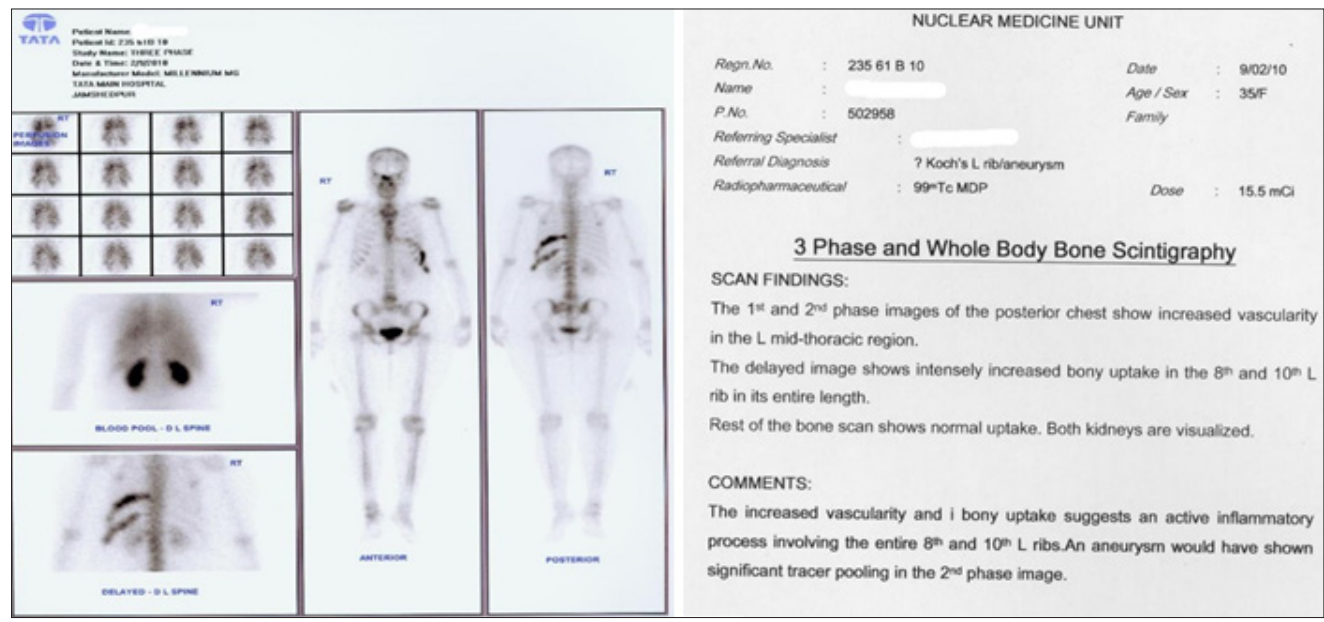

Figure 3.Isotope Bone Scan 99m TC

\section{Investigation}

Routine haematology, biochemistry reports were normal. Chest $\mathrm{X}$ ray showed abnormal trabecular appearance, rarefication on 8th and 10th ribs with clear lung fields (Figure 1). FNAC of the swelling showed no evidence of granulomatous lesion or malignancy. Axial CT scan showed focal expansile lesions of 8th, 10th and 12th ribs on left side with trabeculations and non-enhancing soft tissue densities within (Figure 2), suggestive of aneurysmal bone cyst/ fibrous dysplasia. Pt was further subjected to $99 \mathrm{~m}$ TC whole body bone scan, (Figure 3) which showed increased vascularity over the affected ribs, but absence of significant tracer pooling in phase-II of the scan excluded aneurysmal bone cyst. Rest of the skeleton did not have any occult bony lesions. Hormone screen for thyroid, pituitary, parathyroid and adrenals were in normal range.

\section{Differential Diagnosis}

USG of abdomen, Mammography of breast, Serum calcium, Vit D3 level, parathormone assay, Serum calcitonin levels aided to exclude the D/D of osteomalacia, renal rickets, occult metastasis from abdomen/ breast/ thyroid, Koch's spine/ribs, aneurysmal bone cyst, rare neuro endocrine tumours. Linkage to McCune-Albright syndrome was excluded thro absence of caif au lait spots and normal hormone screen. Absence of classical signs of angina, routine ECG, 2 D echo helped exclude any underlying cardiac cause for left side chest pain. Giant cell tumours are unusual in ribs as they appear near epiphysis. Hence, the diagnosis of Fibrous Dysplasia of ribs (Polyostotic) was arrived at.

\section{Treatment}

With the pt and relatives being alarmed over the radiological findings and continued pain, she was counselled about the rare but benign genetic nature of the disease. She was explained the risks of surgical excision and possible recurrence of such lesions elsewhere, as by now the
Polyostotic nature of the disease was well established. Hence in consultation with her relatives, conservative approach with Calcium, vitamin D3 supplements, graded physical exercises, paracetamol for pain and regular follow up approach was recommended. Bisphosphonates, now advocated for treatment of bone loss was not initiated then, since no long bone was involved. ${ }^{6}$

\section{Outcome and Follow Up}

Pt. has been followed up regularly over last $11 \mathrm{yrs}$ and her lesions have remained static (Figure 4). No new skeletal lesions have been detected elsewhere. The only girl child of the patient has grown up normally and was found not to have any such bony lesion.
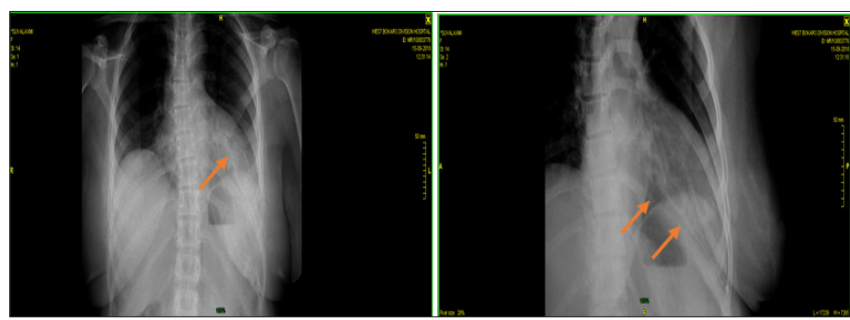

Figure 4.Follow Up Chest X-Ray AP/Left Oblique view after II Years

\section{Discussion}

Lichtenstein and Jaffe first described this condition in 1942. Incidence is around 1 in 1,00,000 cases. ${ }^{1}$ It may involve a single bone (Monostotic type-80\%) or, multiple bones (Polyostotic type-20\%). It manifests clinically in 1st-2nd decade of life around onset of puberty with bone pain, swelling, deformity or pathological fracture or, detected incidentally, confined to one side of the body. ${ }^{1,2}$ The disease is caused by a post conception point mutation in early foetal life, hence not inherited and no vertical transmission is possible. Less than $1 \%$ of cases may undergo malignant transformation to chondrosarcoma, osteosarcoma or fibrosarcoma. ${ }^{3}$ 
McCune-Albright syndrome is linked in 2-3\% of cases, presenting with early puberty and caif au lait spots on one side of body. ${ }^{5}$ This syndrome is caused by a mutation of GNAS gene located in both adrenal glands, responsible for synthesis of $\mathrm{G}$ protein, which in turn, keeps adenylate cyclase enzyme turned on during signal transduction., ${ }^{4,5}$

Axial CT scan is one of the best modality to diagnose the rib involvement, while isotope bone scan helps to differentiate from aneurysmal bone cyst. ${ }^{6}$

The medical management of polyostotic lesions of ribs can be watchful follow up, prevention of de mineralisation of bones with calcium, vitamin D 3 supplements, analgesics, exercise and Bisphosphonates like etidronate, zolendronate, ibandronate, risedronate etc. ${ }^{5,2}$

The surgical management of excision of ribs with chest wall reconstruction or, curettage and bone grafting is usually reserved for cases with pathological fracture or, intractable pain not amenable to NSAID administration. ${ }^{2}$

\section{Learning Points}

Fibrous dysplasia of ribs is a benign bone disorder of post conception genetic mutation aetiology involving GNAS gene. May be observed as abnormal image on chest $x$ ray. Axial CT scan will be best modality in diagnosing the rib abnormality. Analgesics for pain relief and bisphosphonates where bone demineralisation in long bones is present are mainstay of medical management for polyostotic type. Surgical excision is reserved for pathological fracture and intractable pain. McCune-Albright syndrome to be excluded. Rare malignant transformation to osteosarcoma or chondrosarcoma is a feasibility.

Ethical Approval: Not Required

\section{Funding: None}

\section{Conflict of Interest: None}

\section{References}

1. Lietman SA, Levine MA. Fibrous dysplasia. Pediatr Endocrinol Rev. 2013 Jun;10(2):389-96. [Pubmed] [Google Scholar]

2. Stanton RP, Ippolito E, Springfield D, Lindaman $L$, Wientroub S, Leet A. The surgical management of fibrous dysplasia of bone. Orphanet J Rare Dis. 2012;7(1):S1. [Pubmed] [Google Scholar]

3. DiCaprio MR, Enneking WF. Fibrous dysplasia: pathophysiology, evaluation, and treatment. J Bone Joint Surg Am. 2005;87(8):1848-1864. [Pubmed] [Google Scholar]

4. Bousson V, Rey-Jouvin C, Laredo JD, Le Merrer M, Martin-Duverneuil N, Feydy A, Aubert S, Chapurlat $\mathrm{R}$, Orcel P. Fibrous dysplasia and McCune- Albright syndrome: imaging for positive and differential diagnoses, prognosis, and follow-up guidelines. Eur J Radiol. 2014;83(10):1828-42. [Pubmed] [Google Scholar]

5. Chapurlat RD, Orcel P. Fibrous dysplasia of bone and McCune-Albright syndrome. Best Pract Res Clin Rheumatol. 2008 Mar;22(1):55-69. doi: 10.1016/j. berh.2007.11.004. [Pubmed] [Google Scholar]

6. Kransdorf MJ, Moser RP Jr, Gilkey FW. Fibrous dysplasia. RadioGraphics. 1990;10(3):519-537. [Pubmed] [Google Scholar] 